\title{
Bioinspired Mechanisms in Wireless Ad Hoc and Sensor Networks
}

\author{
Anand Paul, ${ }^{1}$ Daniel Bo-Wei Chen, ${ }^{2}$ and Wanmin Wang ${ }^{3}$ \\ ${ }^{1}$ The School of Computer Engineering, Kyungpook National University, Daegu 702-701, Republic of Korea \\ ${ }^{2}$ Department of Electrical Engineering, Princeton University, Princeton, NJ 08544, USA \\ ${ }^{3}$ The School of Electronics and Computer Engineering, Peking University, Shenzhen, Guangdong 518055, China \\ Correspondence should be addressed to Anand Paul; paul.editor@gmail.com
}

Received 19 May 2015; Accepted 27 May 2015

Copyright (C) 2015 Anand Paul et al. This is an open access article distributed under the Creative Commons Attribution License, which permits unrestricted use, distribution, and reproduction in any medium, provided the original work is properly cited.

In the current research inclination, hiring of biological solutions to solve and optimize different aspects of artificial systems' problems has been shaped into an important field with the name of bioinspired computing [1]. The ultimate ideological convergence of scientists to this perfect creation of living beings from the one for finding the relevant traces of problems and their solutions is in fact moving to the best. Tough biological systems exhibit complex, intelligent, and organized behavior, yet they are comprised of simple elements governed by simple rules. It is highly cost effective to hire the complex and networked processing-based simple rules in man-made systems [2]. The real effort that comes in this synergistic solution is finding the similar patterns of issues in artificial (man-made) and real (natural) creation and later their solutions. This invites the interdisciplinary research which is an essential driver for innovation.

Many complete research fields inspired by the constituents of biological system have been established, which have their key roles in optimizing the solution for various problems in nonbiological systems. Among these, a noticeable increase is observed in the interaction of wireless network devices and physical world in numerous ways at various scales from the global Internet down to micro- and nanodevices. Because of the multifaceted nature of these miniature devices solutions from various domains are hired to ameliorate the overall network performance. Wireless sensor network as one of the most emergent miniature technologies with long list of applications in various fabrics of life has been targeted to resolve various issues and to optimize the performance through the solutions of bioinspired domain [3-5].
This special issue aims to emphasize the latest achievements to identify the biological methodologies and their applications to technical solutions in the new research domain of wireless ad hoc and sensor network [6]. These research papers exploit connectionism, social behavior, and emergence of biological systems in various problem solving methods in wireless ad hoc and sensor networks. It may also outline essential challenges, proof of the concept studies with direct and simulated comparison to technical solutions, and mathematical models of biological principles in the same domain.

The research project titled "An Asynchronous Periodic Sequential Pattern Mining Algorithm with Multiple Minimum Item Supports for Ad Hoc Networking" applies a divide-and-conquer strategy to divide the problem of mining asynchronous periodic sequential patterns into a series of mutually disjoint subproblems progressively and then to mine the patterns in such subdatabases. During the process of dividing the database, growing asynchronous periodic sequential patterns and their valid contained segment queues are generated. The algorithm can mine the regular pattern of entity movement trajectory data and predict the future movement.

In the article titled "An ID/Locator Separation-Based Group Mobility Management in Wireless Body Area Network," the sensor node and PMD have globally unique device identifiers (GDIDs). Each GDID contains the information of the associated home network domain. For handover support, each access gateway maintains its home GDID register and its visiting GDID register. In addition, only the coordinator can 
exchange RS/RA messages with PMD instead of each sensor. The numerical analysis shows that the proposed scheme can reduce the registration delay, packet delivery delay, and the handover delay significantly, compared to the existing mobility schemes.

In the article titled "Heuristic Approach to Select Opportunistic Routing Forwarders (HASORF) to Enhance Throughput for Wireless Sensor Networks," the authors analyzed OR schemes in WSNs while focusing on forwarder selection and intuited that a node midway between the previous and next forwarders is a good choice. Inspired by this intuition, they proposed a heuristic forwarder selection scheme, called HASORF, for chain networks. The optimized number of forwarders for a given network is still an open research issue. This research problem is NP-hard and still there is no foundation for analytic thinking; but it is really an interesting problem. Thus the authors intend to dig into this compulsive and strenuous problem in the future.

In the research article titled "A Novel Bioinspired Multiobjective Optimization Algorithm for Designing Wireless Sensor Networks in the Internet of Things," the authors have extended a chaotic ant swarm (CAS). This approach however turns out to be a failure since its outcomes are not even close to the true Pareto front. Then the authors redefined the concept of "neighbors" and "neighbor-selecting" rules and incorporated an "archive-based" approach into the algorithm allowing the resulting Multiobjective CAS (MOCAS) algorithm to converge fast to the true Pareto front with an evenly distributed set of solutions. By testing MOCAS on some wellknown multiobjective optimization problems and comparing the results with that produced by the state-of-the-art peer algorithms MOPSO and NSGA-II, this scheme has proven worthwhile to consider for real-time implementation.

In the research article "Bicriteria Optimization in Wireless Sensor Networks: Link Scheduling and Energy Consumption," the authors investigate the challenging problem of joint optimization on link scheduling and energy consumption for wireless sensor networks. By considering route selection and link scheduling together, we have carried out the analysis of energy consumption and link scheduling for wireless sensor network and formulated it as a bicriteriaobjective integer problem. Considering the specific manyto-one nature of WSNs, we have proposed an efficient optimization scheme based on NSGA-II to solve this problem. In order to ensure the individual validity and fast convergence, the routing tree based representation scheme as well as the corresponding crossover and mutation rules is also designed to satisfy the constraints in the problem. Through extensive simulations, they have demonstrated that their optimization framework based on NSGA-II is quite promising for solving complex multiobjective design models for WSNs.

\section{Acknowledgments}

This work was supported by the IT R\&D program of MSIP/ IITP [10041145, Self-Organized Software platform (SoSp) for Welfare Devices], and it was supported by the Brain Korea 21 Plus project (SW Human Resource Development Program for Supporting Smart Life) funded by Ministry of Education, School of Computer Science and Engineering, Kyungpook National University, Korea (21A20131600005).

Anand Paul

Daniel Bo-Wei Chen

Wanmin Wang

\section{References}

[1] J. Bongard, "Biologically inspired computing," Computer, vol. 42, no. 4, pp. 95-98, 2009.

[2] D. Falko and B. A. Ozgur, "A Survey on bio-inspired networking," Computer Networks, vol. 54, no. 6, pp. 881-900, 2010.

[3] H. J. Rune, Z. Qi, and S. T. Thomas, "Bioinspired principles for large-scale networed sensors systems: an overview," Sensors, vol. 11, no. 4, pp. 4137-2151, 2011.

[4] A. Paul, "Graph based M2M optimization in an IoT environment," in Proceedings of the ACM Conference on Research in Adaptive and Convergent Systems (RACS '13), pp. 45-46, Montreal, Canada, October 2013.

[5] A. Paul, T. A. A. Victoire, and A. E. Jeyakumar, "Particle swarm approach for retiming in VLSI," in Proceedings of 46th IEEE Midwest Symposium on Circuits and Systems (MWSCAS '03), vol. 3, pp. 1532-1545, 2003.

[6] S. S. Iyengar, H. C. Wu, N. Balakrishnan, and S. Y. Chang, "Biologically inspired cooperative routing for wireless mobile sensor networks," IEEE Systems Journal, vol. 1, no. 1, pp. 29-37, 2007. 

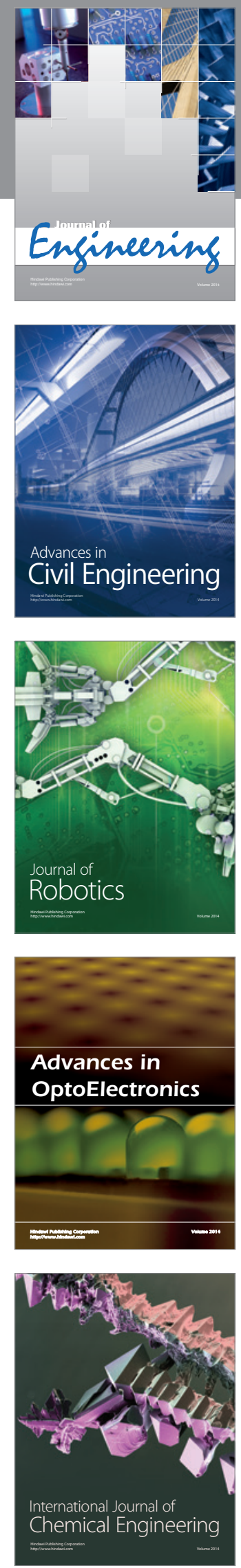

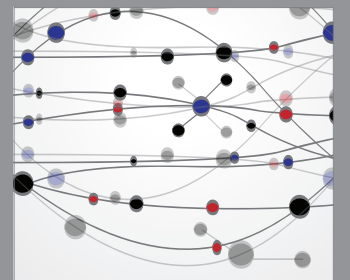

The Scientific World Journal
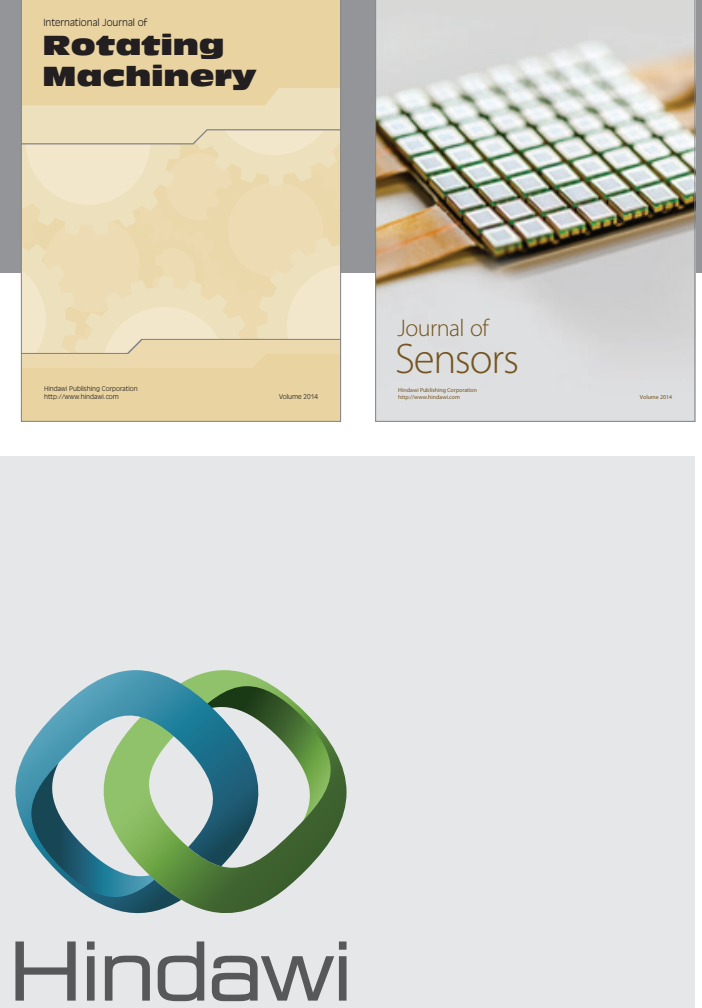

Submit your manuscripts at http://www.hindawi.com
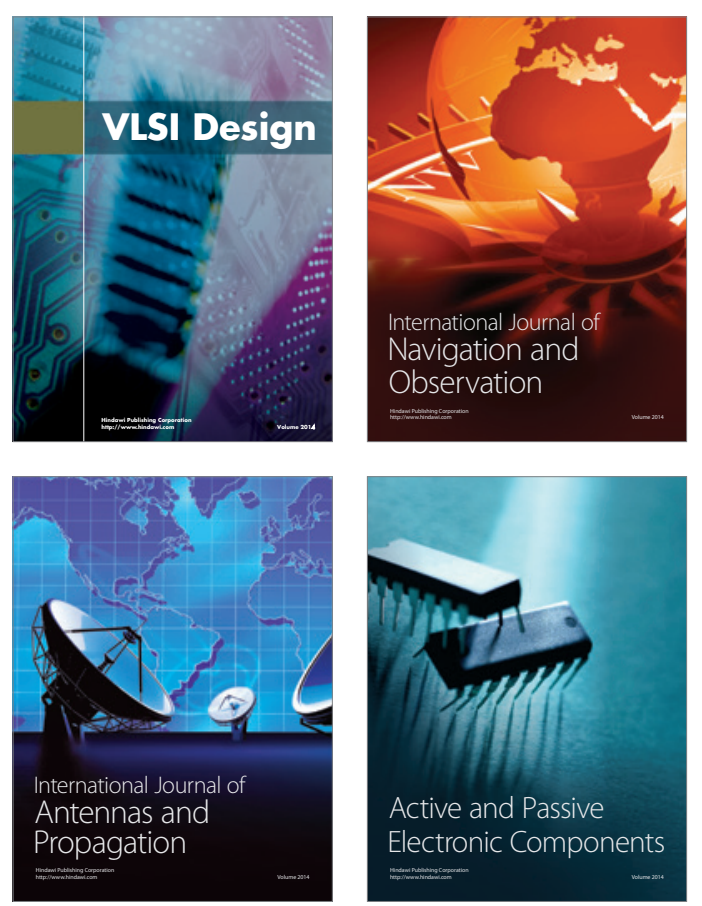
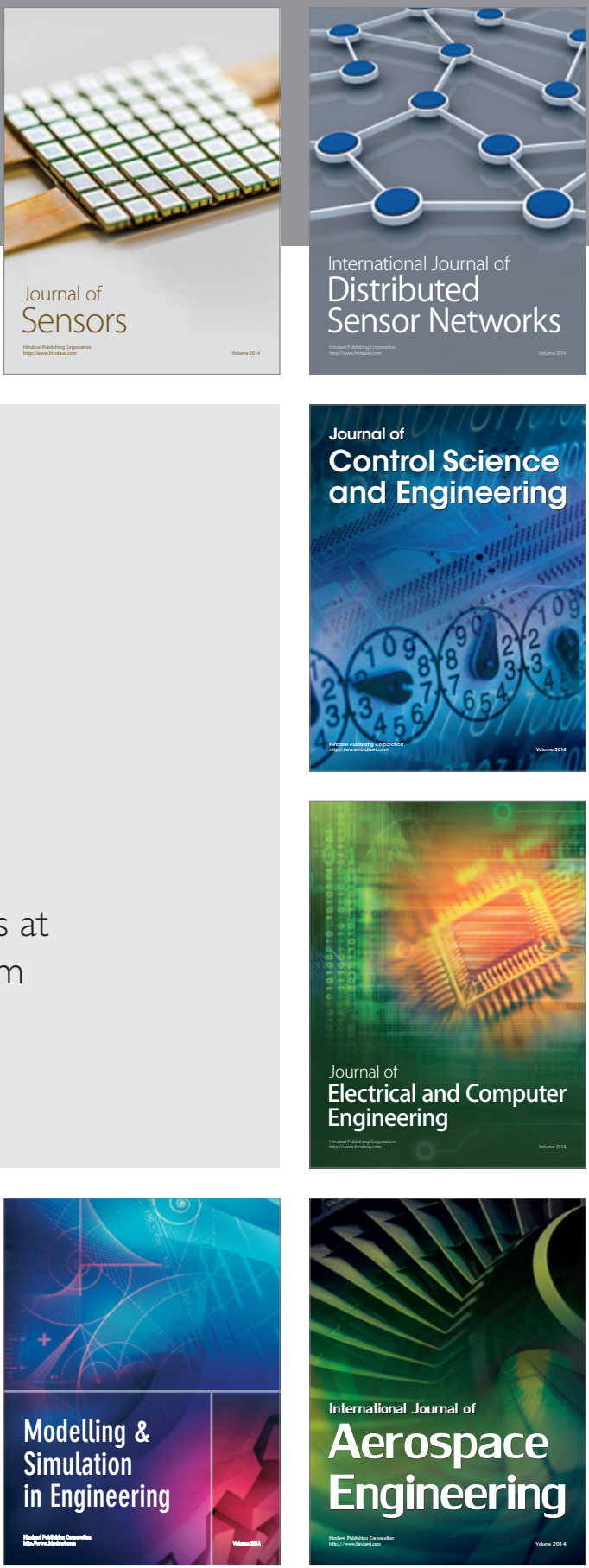

Journal of

Control Science

and Engineering
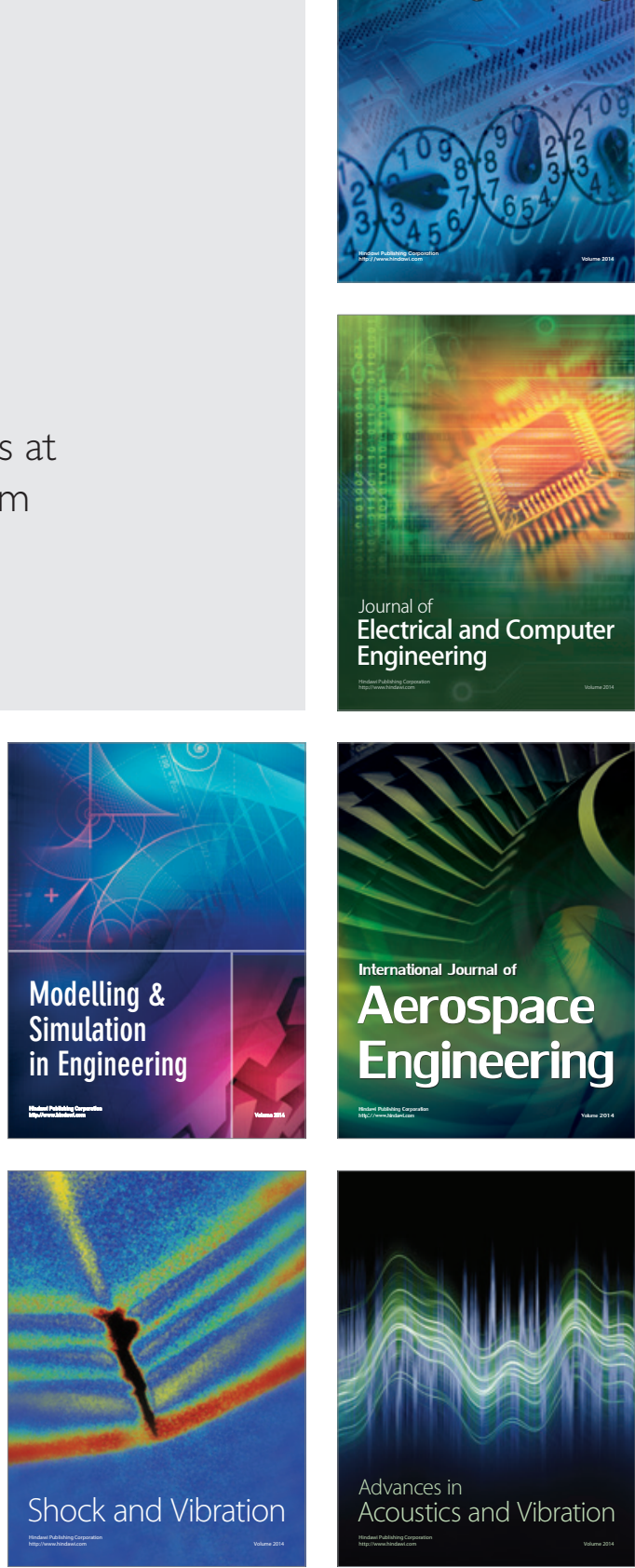